\title{
Counterfactuals and Laws with Violations
}

Cameron Gibbs

Penultimate Draft

Forthcoming in Synthese, 2020, DOI: 10.1007/s11229-020-02740-x.

\begin{abstract}
Evaluating counterfactuals in worlds with deterministic laws poses a puzzle. In a wide array of cases, it does not seem plausible that if a non-actual event were to occur that either the past would be different or that the laws would be different. But it's also difficult to see how we can avoid this result. Some philosophers have argued that we can avoid this dilemma by allowing that a proposition can be a law even though it has violations. On this view, for the relevant cases, the past and the laws would still hold, but the laws would have a violation. In this paper, I raise a problem for the claim that the laws and the past are preserved for all of the relevant counterfactual antecedents. I further argue that this problem undermines motivating the possibility of violations on the grounds that they allow us to hold that the past and the laws are typically counterfactually preserved, even if they are not always preserved.
\end{abstract}

Keywords Laws of Nature $\cdot$ Counterfactuals $\cdot$ Determinism $\cdot$ Violations 
Suppose the laws of nature are deterministic and that I yawned at noon today. Since the laws are deterministic, the laws together with the past guarantee that I yawn at noon today. It seems to follow that on the counterfactual supposition that I do not yawn at noon, either the laws would be different or the past would be different. That is, in a normal context, one of the following is true:

(1) If I had not yawned at noon today, then the past would be different.

(2) If I had not yawned at noon today, then the laws would be different.

But neither of these counterfactuals seems true, and if forced to choose it is not obvious which we should prefer. As a result, a great deal of philosophical work has been devoted to deciding between the two. ${ }^{1}$ However, it would be nice if we could avoid making a choice between two unattractive options. In response, Marc Lange (2000: 73-77), David Braddon-Mitchell (2001), and Boris Kment (2005) and (2014: 213-217) have developed a proposal that promises to do just that. The key is to allow that the laws can have exceptions. On this view, $L$ can be a law even though it has exceptions, that is, even though it is false. ${ }^{2}$ So if I had not yawned, then the past would be the same and the laws would be same. ${ }^{3}$ However, some of the laws would be false, for they would have violations. In exchange for allowing that laws can be violated, we can deny both (1) and (2).

\footnotetext{
${ }^{1}$ See, inter alia, Bennett (1984), Dorr (2016), Goggans (1992), and Lewis (1979).

${ }^{2}$ Braddon-Mitchell (2001: 267) also considers a nearby variant where the laws of nature take the following form: 'the generalization $L$ is part of the best system of laws,' where $L$ can be false.

${ }^{3}$ As Lewis (1979: 462-463) points out, there may be a small transition time before the event in which the past can differ. For simplicity, I set this consideration aside.
} 
As plausible as this line of thought might seem, I will argue that it runs into serious difficulties. In particular, we'll see a problem raised by iterated counterfactuals, that is, counterfactuals of the form 'if $P$ were true, then if $Q$ were true, then $R$ would be true.' But before considering the problem, we will need to lay out some preliminaries in the next section.

\section{Preliminaries}

Orthodoxy accepts the following schema:

Factive Laws Necessarily, if it is a law that $P$, then $P$.

But the believer in violations denies Factive Laws, allowing that laws can be violated. For simplicity, I will make the standard assumption that laws take the form of exceptionless regularities, so that a violation renders a law false. Thus, the believer in violations accepts:

Violations For some $P$, it is a law that $P$, and possibly, $\sim P$ and it is a law that $P .{ }^{4}$

We'll be working with the assumption that the laws are deterministic. I'm understanding deterministic laws in the following way: a set of laws $L$ is deterministic iff any two possible worlds at which the laws $L$ are true that are intrinsically the same at some interval of time are intrinsically the same at all times. ${ }^{5}$ It follows that if the laws $L$ are deterministic, then any two worlds where

\footnotetext{
${ }^{4}$ The believer in Violations may strengthen this by holding that all laws could be violated, but that is an extra commitment that I do not assume the believer in Violations must take on.

${ }^{5}$ This way of defining deterministic laws is similar to the definitions found in Lewis (1986a) and Dorr (2016).
} 
the laws $L$ are true that intrinsically differ at some interval of time, must intrinsically differ at all other times. In the case of our example involving yawns in (1) and (2), the idea is that my yawning at noon is an intrinsic feature of the world at noon. If the laws are deterministic, then in any possible world with the same laws, where I do not yawn at noon, the past must differ in some intrinsic way from the actual past.

Why accept Violations? The classic motivation appeals to actual examples of violated laws of nature. ${ }^{6}$ For example, if it is a law that all ravens are black, then albino ravens constitute a violation of this law. However, I wish to set aside this motivation and focus on a different motivation for Violations, namely, the relationship between laws and counterfactuals. This alternative motivation is of particular interest given that many philosophers remain unconvinced by the alleged examples of violated laws, for there are many ways of handling these examples without appealing to violated laws. On the one hand, one might simply deny that the violated regularity is a law. In the example above, one might deny that it is a law that all ravens are black. Alternatively, one might hold that the law should be understood in such a way that is consistent with the alleged violations. For example, one might hold that the law involving ravens is best formulated as a generic: generically, ravens are black. One might instead take the law to be a hedged law: ceteris paribus, all ravens are black. On both of these approaches, the law is consistent with non-black ravens, and so is not violated. ${ }^{7}$ In light of these ways of handling the potential examples without appealing to violated laws, it's worth investigating other ways of motivating Violations.

\footnotetext{
6 The classic case is Cartwright (1980).

${ }^{7}$ For a useful recent discussion, see Roberts (2008: 48-50).
} 
As I mentioned above, my interest is in whether Violations can be motivated on the basis of the relationship between laws and counterfactuals. The thought is that Violations is justified on the grounds that it allows us to deny both (1) and (2). More generally, I'm interested in the claim that we should accept Violations because it allows us to accept the instances of the following schemas:

(3) If $P$ were true, then the past would be the same.

(4) If $P$ were true, then the laws would be the same. ${ }^{8}$

It is important that we cannot substitute nomologically impossible propositions for $P$. Plausibly, if a particle were to move faster than the speed of light, then different laws would hold. The defender of Violations does not need to reject that. ${ }^{9}$ However, care needs to be taken in how we understand nomologically impossible propositions. Given Violations, a law can be false, so a proposition can be nomologically possible even if it is inconsistent with the laws of nature, for that proposition could describe the occurrence of a violation. Rather, we should follow Kment (2006: 289-291) and define a proposition as nomologically possible just in case it is consistent, not with the truth of the laws, but with all of the laws still being laws.

A key part of my argument involves iterating counterfactuals, that is, counterfactuals of the form 'if $P$ were true, then if $Q$ were true, then $R$ would be true.' (3) and (4) do not extend to

\footnotetext{
${ }^{8}$ For independent reasons, Lange (2000: 177) does not think these sorts of counterfactuals are always true, but near enough to being 'correct' to assert. I set aside this qualification in what follows.

9 There are also so-called 'back-tracking' contexts where it is uncontroversial that the past is not preserved when evaluating counterfactuals. For present purposes, we set aside contexts that are uncontroversially back-tracking. For more discussion, see Lewis (1979: 456-458).
} 
iterated counterfactuals of this form, but this is an extremely natural generalization of (3) and (4). If you do not think we have counterfactual power over the laws, then you also shouldn't think that were something nomologically possible to occur, then we would have counterfactual power over the laws. For example, suppose you hold that were I to raise my hand, then the laws would be the same. You should then also hold that were you to raise your hand, then were I to raise my hand, then the laws would be the same. By parallel reasoning, the same applies to counterfactually preserving the past. So the defender of Violations should also embrace the generalization to iterated nomologically possible counterfactual antecedents. A simple way of accommodating this is for the defender of Violations to further hold that the instances of (3) and (4) are nomologically necessary, and that nomological necessity obeys a modal logic at least as strong as $\mathrm{S} 4$, so that if $P$ is necessary, then necessarily $P$ is necessary. ${ }^{10}$ This guarantees that every instance of (3) and (4) is true in each nomological possibility, as well as in every iteration of nomological possibilities. This will be enough to secure the iterated counterfactuals for as we iterate nomologically possible antecedents, at every step of the way (3) and (4) will hold and so the laws and the past will always be held fixed. In light of my argument, the defender of Violations may weaken their view, and reject the iterated counterfactuals. Later I will consider and argue against such a proposal. ${ }^{11}$

If every instance of schemas (3) and (4) is nomologically necessary, then say that the laws and the past are counterfactually robust. According to the defender of Violations, by allowing for the possibility of violations we can hold that the laws and the past are counterfactually robust. This allows us to preserve the intuitive idea that for a wide array of counterfactual antecedents the laws would still be the laws and the past would be the same. I want to limit our focus to this particular

\footnotetext{
${ }^{10}$ The defender of Violations may wish to further strengthen their view and hold that (3) and (4) are metaphysically necessary, rather than simply nomologically necessary. However, we will only consider nomologically possible antecedents, so this extra strengthening is unnecessary.

${ }^{11}$ I thank an anonymous referee for helpful comments on this issue.
} 
potential source of justification for Violations. Of course, one might accept Violations on other grounds, in which case one is free to appeal to the possibility of violations in evaluating counterfactuals. But even then, the argument of this paper shows that there are surprising constraints on how violations can be used to evaluate counterfactuals.

The notion of an instance of a law of nature plays an essential role in my argument. However, I'll need a specific sense of this notion. We sometimes speak of instances of a law of nature in such a way where any event or state of affairs that is consistent with that law is an instance of the law. On this understanding, if it is a law that all ravens are black, then a black raven is an instance, as well as a non-black, non-raven. This understanding is often at play when discussing how laws of nature are confirmed.

However, I require a more discriminating sense of an instance of a law of nature, which we could call the positive instances. This is the sense in which it makes sense to speak of laws of nature without any instances. If we have the law that all $F \mathrm{~s}$ are $G \mathrm{~s}$, then I take something being both an $F$ and a $G$ to be an instance of this law, but not something being neither $F$ nor $G .^{12}$ Of course, laws might take other forms as well. For example, many laws are best thought of as equations that relate quantities rather than universal generalizations. In this case, I take the instances of this law to be events involving the relevant values of the quantities being coinstantiated in the way the equation says they are. By contrast, I do not count as instances events where no values of the quantities are instantiated at all. For example, in the case of the law $F=M A$, there are no instances of this law in locations where mass is not instantiated.

\footnotetext{
12 Note that since the claim that all $F_{\mathrm{S}}$ are $G \mathrm{~s}$ is logically equivalent to the claim that all non- $G \mathrm{~s}$ are non- $F_{\mathrm{S}}$, I require a fairly fine-grained notion of laws of nature, so that the law that all $F$ s are $G$ s is not identical with the law that all non- $G$ s are non- $F \mathrm{~s}$. Those inclined to reject this assumption should replace my talk of laws with talk of law statements, as the statement that all $F \mathrm{~s}$ are $G \mathrm{~s}$ is not identical with the statement that all non- $G$ s are non- $F \mathrm{~s}$. As far as I can see, my argument is not affected by this substitution.
} 
In contrast to instances are violations. An event is a violation of a law $L$ just in case in every world where $L$ is a law and the event obtains, $L$ is false. If Factive Laws is true, then there is no world that contains a violation of a law. However, Violations implies that there are laws that could have violations.

In the next section, I argue that by the lights of the believer in Violations the laws and the past are not counterfactually robust. One might attempt to justify Violations by appealing to the instances of (3) and (4) that do hold, even if they do not hold in full generality. However, in a later section, I will argue against this alternative way of justifying Violations. ${ }^{13}$

\section{Iterating Counterfactuals}

It will be helpful to begin with a promising, but unsuccessful, counterexample to the claim that the laws and the past are counterfactually robust. According to the defender of Violations, the counterfactual robustness of the past and the laws implies that when we consider a counterfactual supposition that is not in fact true, then we go to the nearest world where we replace an instance of some law $L$ with a violation of that law. Plausibly, for every instance of $L$, there is some counterfactual supposition $P$ such that were $P$ to be the case, then a violation of $L$ would obtain instead of that instance. Suppose we conjoined together each of these suppositions. Call this The Conjunction. If The Conjunction were true, then it seems that the result would be a world where every instance of $L$ is replaced with a violation. In such a world, surely $L$ is no longer a law. So

\footnotetext{
${ }^{13}$ While Braddon-Mitchell (2001: 270-272), Kment (2014: 216), and Lange (2000: 76-77) do not commit themselves to the claim that the laws and the past are counterfactually robust in full generality, they do aim to support Violations on the grounds that it allows us to hold that the laws and the past are at least generally counterfactually preserved.
} 
we have a failure of (4); there is a supposition that counterfactually implies that there would be different laws. ${ }^{14}$

One way of rejecting this counterexample is by allowing that $L$ could be a law even if $L$ has no instances and many violations. Later I'll consider this view and argue against it. Setting this response aside, there is a deeper problem for the argument.

The real trouble with the counterexample is that there is no guarantee that The Conjunction counterfactually implies a world where $L$ has many violations and no instances. First, even though each antecedent individually counterfactually implies that a violation would obtain, it does not follow that their conjunction counterfactually implies that each of those violations would obtain. It is invalid to infer from 'if $A_{1}$ were true, then $B_{1}$ would be true' ... 'if $A_{n}$ were true, then $B_{n}$ would be true' that 'if $A_{1}$, and .., $A_{\mathrm{n}}$ were true, then $B_{1}$, and $\ldots, B_{\mathrm{n}}$ would be true.' Here's a simple example to see this. Consider a game of basketball. If Jane had been put in play, then Jane would score 20 points. If Joe had been put in play, then Joe would have scored 20 points. But Jane and Joe do not play well together, so if they both had been put in play, then neither of them would have scored 20 points. So The Conjunction may not counterfactually imply all of the violations.

Second, The Conjunction may counterfactually imply further instances of the law, in which case we will not have a world where the law has no instances and many violations. We could add to The Conjunction in order to rule out these cases, but the trouble is that as we add to The Conjunction we are not guaranteed that the antecedent is nomologically possible, in which case we will no longer have a counterexample to the counterfactual robustness of the laws. As a result,

\footnotetext{
${ }^{14}$ Note that if The Conjunction counterfactually implies that $L$ would no longer be a law, that does not show that The Conjunction is nomologically impossible. It could be that if the past were different then The Conjunction could be true and $L$ would still be a law.
} 
it is hard to see how to make this counterexample in a way that would be convincing to the defender of Violations.

So this counterexample to the counterfactual robustness of the laws and the past is not convincing. However, there is a similar, but more sophisticated, counterexample that is harder to avoid. Instead of conjoining together several counterfactual suppositions into a single supposition, we can raise a problem by iterating those suppositions. In this way of raising the problem, we first consider a counterfactual supposition, and in that supposition we consider another counterfactual supposition, and so on. As we'll see, making the argument this way avoids the problems that faced the simpler counterexample.

Consider a law $L$ with no violations, and let's assume that there is a first moment $t$ when $L$ has an instance (later I'll argue that we can dispense with this assumption). While $L$ has no violations, the defender of Violations will hold that $L$ could have violations. So let's consider a nomologically possible antecedent $A_{1}$ that counterfactually implies, by the lights of the believer in violations, that the instance of $L$ at $t$ would not obtain, but rather a violation $V_{l}$ of $L$ would obtain at $t .{ }^{15}$ The believer in Violations accepts the instances of (3) and (4), so they hold that in the nearest world in which $A_{l}$ obtains the same laws hold, the same past holds, and violation $V_{l}$ obtains.

In the world where $A_{1}$ is true, consider the next instance of $L$, and let $A_{2}$ be a nomologically possible antecedent that counterfactually implies that the instance of $L$ would not obtain, but rather a violation $V_{2}$ of $L$ would obtain at that time. Again, the believer in Violations accepts the instances

\footnotetext{
${ }^{15}$ Are we guaranteed that there is such an antecedent? If $A_{l}$ simply says that the instance of $L$ doesn't obtain and (3) and (4) hold, then we are guaranteed a violation, given the assumption of determinism, but we aren't guaranteed that it is $L$ that is violated rather than some other law. However, we can add to the antecedent to ensure that at least $L$ is violated. If $L$ is not violated because some other law $L^{*}$ is violated, then we can add to the antecedent so that $L^{*}$ is not violated at the relevant time and place.
} 
of (3) and (4), so in the nearest world the same laws hold and the past is the same. Importantly, since the past is the same, the past contains the earlier violation $V_{l}$.

We can repeat this process indefinitely and if each instance of (3) and (4) holds throughout the series, then eventually we will be in a world where the law has no instances and is violated at every turn. But this seems absurd. As I will argue shortly, even if a law can have violations, surely it cannot be a law when the world only violates it and never obeys it. But if we deny the possibility of a law that has no instances and many violations then we must deny an instance of either (3) or (4) along the way.

So after a series of nomological possibilities, we've come upon a world where an instance of either (3) or (4) fails. Assuming that a logic at least as strong as S4 governs nomological possibility, this final world is itself nomologically possible. Moreover, this series gives rise to an iterated counterfactual in the actual world. Suppose for a particular case we have a series of nomologically possible counterfactual suppositions $A_{l}, \ldots, A_{n}$. For concreteness, suppose that the defender of Violations holds that once we reach $A_{n}$, then the relevant instance of (3) fails. We will then have the following iterated counterfactual:

(5) If $A_{1}$ were true, then if $A_{2}$ were true, ..., then if $A_{n}$ were true, then the past would be different.

Importantly, (5) is true in the actual world. But recall that if someone accepts (3), then they should also accept generalizing (3) to counterfactuals with iterated antecedents, such as:

(6) If $A_{1}$ were true, then if $A_{2}$ were true, ..., then if $A_{n}$ were true, then the past would be the same. 
But clearly, we must reject (6) if we accept (5), posing a problem for the defender of Violations.

\section{Challenging the Assumptions}

This argument made use of several assumed premises. Can the argument be evaded by rejecting these premises? In this section, I argue 'no.' In the case of some of the premises, I will argue that we have strong grounds for accepting them. In the case of other premises, I will argue that they are dispensable. Even if we deny these premises, there are nearby arguments for the same conclusion.

A key assumption of the argument is that it is not possible for there to be a law with no instances and many violations. To be clear, the objection is not to laws that merely have no instances; rather, the objection is to laws that are frequently violated and never instanced. Normal cases of uninstantiated laws are quite different. These are typically cases involving a law that governs the outcome of an interaction or a process, but where the interaction or process never occurs. ${ }^{16}$ For example, consider a law that governs the result of the interaction between two kinds of particles in a world where those particles never interact. In these cases, the law has no instances because it never has an opportunity to have instances. By contrast, a law with no instances but many violations has many opportunities to have instances; every violation is an opportunity to have an instance.

So why hold that there could not be laws with no instances and many violations? First, note that Braddon-Mitchell's (2001) particular account of laws with violations doesn't allow for laws with no instances and many violations. Braddon-Mitchell accepts a Humean view; a version

\footnotetext{
16 See Tooley (1977) and Carroll (1994: 60-80) for more on these cases.
} 
of the best-systems account of laws. On his view, the laws are the regularities that show up in the system that best systematizes all the facts. The best system is the system that best balances strength, simplicity, and accuracy. His view allows for violations, for a small cost in accuracy may allow for great gain in strength and simplicity. But if a law is only violated and never obeyed, then adding it to a system of laws will be a great cost in accuracy with no gain in strength or simplicity. Setting aside the specifics of Braddon-Mitchell's view, it seems unlikely that any Humean would want to allow for laws with no instances and many violations. Humeans are united in holding that the laws somehow describe the non-nomic facts. But a law that is never instanced and frequently violated would completely fail to describe the non-nomic facts.

Even setting aside the Humean view of laws, we have reason to reject laws that are never instanced and frequently violated. For what would justify calling a proposition a law in a world like this? This is particularly pressing because a law like this would do an extremely poor job of playing the theoretical roles associated with laws. First, laws should be explanatory. But a constantly violated law with no instances would not have any explanatory power; nothing that occurred in the world would be explained by the law. Now, it's true that a law like this would be similar to any other uninstantiated law in that both would vacuously explain all of their instances, since they have no instances. ${ }^{17}$ But there is a crucial difference between the two kinds of laws. Normal uninstantiated laws simply lack an opportunity to have any instances, and by extension, any opportunity to explain anything. However, laws that are frequently violated but lack instances, have many opportunities to explain. Every violation is a missed opportunity to instead explain an instance. So we could say that the way in which these laws fail to satisfy the theoretical role of being explanatory is by failing to explain anything given many opportunities to do so.

\footnotetext{
${ }^{17}$ I am grateful to a referee for pointing this out.
} 
Second, laws should license predictions. But these violated laws would not license even remotely reliable predictions about the future. In such a world, the law would massively mislead us about what will happen. Now, it's true that we could be justified in using an inference even if that inference might sometimes lead us astray. But in this case, the law would not merely occasionally lead us astray, it would lead us into systematic error. One would not be justified in using a law like this to make predictions.

Finally, laws should be confirmable. We would never be able to know that these violated laws were laws of nature, since they wouldn't be confirmable in any way. Without positive instances or explanatory power, we could never be justified in believing in such a law.

It might be negotiable how much of these theoretical roles a proposition needs to satisfy in order to be a law of nature. For example, some non-Humeans might be comfortable with laws that cannot, in principle, be confirmed. But if a proposition systematically fails at every theoretical role associated with laws of nature, then we are not justified in calling that proposition a 'law.'

It's worth turning to focus specifically on non-Humeans. Non-Humeans hold that whether or not a proposition is a law fails to supervene on the non-nomic facts. Since the laws float free from the non-nomic facts, this view seems to have the resources to make sense of a law that has no instances and many violations. However, there is still reason for the non-Humean to deny this possibility. The non-Humean posits some ontology or ideology over and above the non-nomic facts in order to account for the laws of nature. ${ }^{18}$ Think of these posits as lawmakers, in the sense that they are intended to account for the laws of nature. But the non-Humean takes on the burden of demonstrating that their posit deserves the name 'lawmaker' by adequately accounting for the

\footnotetext{
${ }^{18}$ For example, one might understand laws in terms of a nomic relation between universals, as in Armstrong (1983), Dretske (1977), and Tooley (1977); or in terms of essential dispositions, as in Bird (2007); or simply take them to be primitive, as in Carroll (1994) and Maudlin (2007).
} 
laws of nature. The best way to make the case that these posits deserve the name of 'lawmaker' is by showing that the posit plays the right theoretical role. However, in a world where a law of nature fails to be explanatory, confirmable, or license future predictions, it's hard to see why these posits deserve the name 'lawmaker.'

A further worry is that allowing the possibility of laws that have no instances and many violations undercuts an important motivation for non-Humean accounts of laws. Many nonHumeans justify their account of laws by arguing that this account of laws affords a way of making sense of the rationality of induction. ${ }^{19}$ The basic idea is that the best explanation of an observed regularity involves a non-Humean law, which in turn guarantees the reliability of projecting this regularity in the future. But if it is possible for a law to have no instances and many violations, then an observed regularity could simply be a series of violations, with no law to explain them.

A final worry for the non-Humean is that allowing the possibility of laws with no instances and many violations threatens to raise more problems for the view. Non-Humeans face a challenge in accounting for the relationship between their posited lawmakers and the regularities among the non-nomic facts. The simplest way of accounting for the relationship is to associate a posited entity with a regularity and hold that the existence of the entity entails the regularity. For example, Armstrong (1983), Dretske (1977), and Tooley (1977) account for laws in terms of a relation between universals. On this view, a natural way to account for the relationship between lawmakers and regularities is to hold that the universal $F$ bearing this lawlike relation to $G$ entails the regularity that all Fs are Gs. If Factive Laws is given up, then this entailment does not hold. So the relationship between lawmakers and regularities will have to be accounted for in other terms. But matters are far worse if the non-Humean admits the possibility of a law with no instances and

\footnotetext{
${ }^{19}$ For defense of this point, see Armstrong (1983), Foster (1982-1983), Hildebrand (2018), Huemer (2009), and Tooley (2011). For criticism, see Beebee (2011).
} 
many violations, for then there is no guarantee of any interesting relationship between lawmakers and regularities at all. So the non-Humean also has strong reason to deny the possibility of a law with no instances and many violations.

Another assumption of the argument is that as violations are added to the series of worlds, the added violations do not counterfactually imply a further instance of the violated law after the occurrence of the violation. By rejecting this assumption, one could argue that we will never arrive at a world where the law lacks any instances; on this view, as we move along the series there will always be further instances of the law. However, even without the assumption, the argument still works. We can change the antecedents of the counterfactuals in such a way that they do not counterfactually imply further instances of the law. For example, we could change the antecedent from 'if $A_{l}$ were true' to 'if $A_{l}$ were true and there were no new instances of $L$.' These antecedents would not counterfactually imply a further instance of the law, and these antecedents are all nomologically possible.

I also assumed that a violation's obtaining does not counterfactually imply that a different violation would not obtain. By denying this assumption, one might reject my argument on the grounds that there would never be very many violations, since adding a violation would counterfactually imply that a different violation would not obtain. Since the defender of violations holds that the past is held fixed, a violation cannot counterfactually imply that a past violation would not obtain. I constructed the argument in such a way that we move forward in time, so we will always keep the same violations in the past.

However, what about violations that occur at the same time? Suppose $V_{n}$ and $V_{m}$ are violations at the same time, but are such that if one occurred then the other would not. In that case, we cannot add one violation without removing the other. However, as long as it is nomologically 
possible for both violations to obtain, we can avoid the issue by using a single antecedent to counterfactually imply that both violations would obtain. If it turns out that it is not nomologically possible for both violations to obtain, then we can change our antecedent so that one of the violations obtains and no other instances of the law obtains at that time.

Finally, what if one of the violations counterfactually implies that the future violation would not occur? ${ }^{20}$ Again, suppose that we have violations $V_{n}$ and $V_{m}$, but this time $V_{n}$ occurs before $V_{m}$ and counterfactually implies that $V_{m}$ would not obtain. However, we can still ask what would happen were one of the later counterfactual antecedents to obtain. Given the truth of Determinism, and the commitment to holding fixed the laws and the past, a violation must still occur. Perhaps, the particular violation $V_{m}$ would not obtain, given the earlier violation $V_{n}$. But some violation or other must obtain. ${ }^{21}$ The argument can then run through the same.

Finally, I made several assumptions about the instances of the law $L$. I assumed that there is a first instance, a last instance, and that the instances are not dense, in that it is not the case that between any two instances there is another instance. But these assumptions may be false.

One challenge for avoiding my argument by rejecting these assumptions is that even if these assumptions don't hold for all laws, it is plausible that they hold for some laws. After all, any law that only has a finite number of instances will have first and last instances, and the law's instances will not be dense. As long as the argument can run for even a single law, that is enough to show that the laws and the past are not counterfactually robust.

However, a deeper challenge to this response is that even if these assumptions are rejected, we can formulate nearby arguments for a similar conclusion. Let us consider these assumptions

\footnotetext{
${ }^{20}$ I thank an anonymous referee for bringing this possibility to my attention.

${ }^{21}$ Perhaps this doesn't guarantee that the right law is violated, maybe some other law would be violated instead. But, as noted in footnote 14, we can avoid this problem by changing the antecedent to also state that this other law is not violated.
} 
in turn. First, it may be that there is no first instance, but rather an infinite series of instances of the law in the past. However, even if the assumption is false, it may still be nomologically possible for $L$ to have a first instance, in which case we can run the argument by appealing to these worlds. To obtain the required iterated counterfactual, we can let the first antecedent be if $L$ were to have a first instance.' Alternatively, we can sidestep the need to appeal to a first instance of $L$. If we can't appeal to a first instance, then we can appeal to a given instance and run the argument for every instance after that. This would give us a world where $L$ had no violations up to a particular time, but had no instances and only violations after that time. Moreover, we can run this argument for any given instance; so for any instance, there is a world where $L$ is only violated and never obeyed after that instance. While perhaps not as bad as a world where a law is never instanced and frequently violated, these laws still have many of the same drawbacks. The reasons given for rejecting a law that is never instanced and frequently violated would seem to carry over to these cases as well. It does not seem likely that laws like this will always belong to the Humean's best system of regularities. It also does not seem likely that laws like this will always play the theoretical roles of being explanatory, licensing predictions, and being confirmable. Finally, it does not seem likely that laws like this will always cohere with non-Humean's conception of laws.

Further, there's a plausible argument that if for any instance of $L$ there's a world where $L$ is always violated after that instance, then there's also a world where $L$ is always violated full stop. The argument is that to reject the final possibility while accepting the former possibilities would amount to an arbitrary gap in the space of possibilities; the former possibilities form a natural sequence that leads to the final possibility. But, as many philosophers have pointed out, one of our most basic intuitions about the space of possibilities is that it lacks arbitrary gaps of this sort. ${ }^{22}$

\footnotetext{
22 As Dorr (2004: 42) puts it: "That there are no such arbitrary limitations on the space of possibilities is one of our firmest modal intuitions.” Lewis (1986b: 86-92), Bricker (1991), and Chalmers (1996: 137) concur.
} 
In order to avoid arbitrary gaps in the space of possibilities, if we accept that for any instance of $L$, there's a world where $L$ is always violated after that instance, then we should also accept a world where $L$ is always violated full stop.

Let's now turn to the assumption that $L$ has a final instance. This assumption may be false, for $L$ might have infinitely many instances in the future. While the way I originally formulated the argument appealed to a final instance, this is a dispensable assumption. If there is no final instance of $L$, then we won't have an embedded counterfactual of the form 'if $A_{l}$ were true, then if $A_{2}$ were true, ..., then if $A_{n}$ were true, then $P$ would be true,' for there is no final antecedent $A_{n}$. However, the argument still has force even if there is no last instance, because we can have an embedded counterfactual with infinitely many embedded antecedents. We can certainly understand counterfactuals with infinitely many embeddings. For example, suppose I have a quantity of infinitely divisible matter. We can clearly see that the following counterfactual is true:

(7) If I were to remove half of the matter, then if I were to remove a quarter of the matter, then if I were to remove an eighth of the matter, ..., then there would be no matter left.

Since we do not have any difficulty in seeing that this counterfactual is true, we do not have any general difficulty with understanding a counterfactual with infinitely many embedded antecedents. So even if $L$ has no final instance the argument can still go through. If we accept the schemas (3) and (4) for every instance, then we will end up with a world where $L$ is a law, but where there are no instances and many violations of $L$. If we reject this world, then we must reject an instance of either (3) or (4). 
Let's now consider the final assumption. In my presentation of the argument, I assumed that the instances of $L$ are not dense; in other words, I assumed that it was not the case that between any two instances of $L$ there is another instance. One way of responding to this problem would be to formulate the argument in terms of a counterfactual with embedded antecedents, but where the embedded antecedents are densely ordered. This sort of counterfactual will have the following form: if $A_{1}$ were true, ..., then if $A_{n}$ were true, ..., then if $A_{m}$ were true,..., then $B$ would be true. But it's not clear that we can understand these constructions or how they are assigned truth conditions, so I don't wish to rest my argument on constructions like these.

However, there is a way of formulating the argument that avoids this problem. If the instances are densely ordered, then we can instead appeal to regions of spacetime that contain these instances. If we take regions that contain more than one instance, then we can take a series of spacetime regions that are not densely ordered. ${ }^{23}$ Once we have such a series of regions, we can let our counterfactual antecedents be that those instances do not obtain in the regions. More exactly, for every region, we associate a corresponding counterfactual antecedent that says that the instances in the region do not obtain. Given that the same past and the same laws hold, this will require that a violation obtains in the region without any of the instances. We can then run the argument using these antecedents instead.

\section{How Serious is this Problem?}

\footnotetext{
${ }^{23}$ To get a sense for how this could work, we can think of the structure of the densely-ordered instances along the lines of the positive rational numbers, for between any two rational numbers there is another. We can now take a nondensely-ordered series of regions that contain these instances. This could be done by thinking of the regions as collections of rational numbers. For example, we have a collection of all rational numbers greater than 0 and less than 1 , a collection of all rational numbers at least as great as 1 but less than 2 , and so on. Thought of in this way, there is no challenge to taking a non-densely-ordered series of spacetime regions that contains the instances of the law.
} 
We have seen a counterexample to the claim that the laws and the past are counterfactually robust. So the argument for Violations from the counterfactual robustness of the laws and the past is unsuccessful. However, the defender of Violations might respond with the following line of thought: even if the laws and the past are not always counterfactually preserved, they are preserved for most counterfactual antecedents. Further, the best way to account for this is by allowing for the possibility of violations. So even if we deny that the past and the laws are counterfactually robust, we can still justify Violations on the grounds that the laws and the past are usually, if not always, counterfactually preserved.

The central challenge for this response is to explain why we should hold that the laws and the past are generally counterfactually preserved if they are not always counterfactually preserved. The natural justification is to appeal to our intuitive judgments about the truth of the relevant counterfactuals. To fix ideas, let's suppose that this response appeals to our intuitive judgments about the instances of (3) and (4), but does not generalize this to counterfactuals with embedded antecedents. It will be useful to have some terminology. Recall that the laws and the past are counterfactually robust if every instance of (3) and (4) is nomologically necessary. If every instance of (3) and (4) are true, let's say that the laws and the past are weakly counterfactually robust. If the laws and the past are counterfactually robust, then they are weakly counterfactually robust, but the reverse does not hold. What are the prospects for justifying Violations by appealing to the claim that the laws and the past are weakly counterfactually robust?

This route to defending Violations still faces several problems. First, the intuitions in favor of the instances of (3) and (4) that lack embedded antecedents generalize to counterfactuals that do have embedded antecedents. To use an example from before, suppose our arms are not raised. If one thinks that if I were to raise my arm, then the past would be the same, then they should also 
think that if you were to raise your arm, then if I were to raise my arm, then the past would be the same. It would be arbitrary to accept the former, but not the latter. Further, the parallel thought holds for the laws. But the argument in the previous section demonstrates that after a sufficient number of embedded counterfactual antecedents, either the past or the laws will no longer be preserved. Since our intuitions in support of the counterfactuals that do not have embedded antecedents generalize to the counterfactuals that do have embedded antecedents, we have an argument for the conclusion that these intuitions lead us into falsity. This gives us reason to regard these intuitions with suspicion.

A further problem arises if there is a final world in the series before the world where either the past or the laws are different. The trouble is that in this final world, a counterfactual of the following form is true: if $A_{n}$ were true, then either the laws or the past would be different. But this sort of counterfactual without an embedded antecedent is exactly the sort of counterfactual that we are supposed to have a strong intuition against. While counterfactuals like these seem to be false in the actual world, it is equally plausible that they are false in every nomologically possible world. This also threatens to undermine our reasons for thinking these counterfactuals are false in the actual world. If not, then the defender of Violations takes on the burden of explaining why our intuitions against these counterfactuals do not carry over to these other possible cases.

The defender of violations might object that in the series of worlds there are borderline cases regarding whether the past or the laws are counterfactually preserved, and so it is not determinate which world is the final world in the series. However, this response would seem to require holding that there are worlds where the relevant counterfactuals are indeterminate. This is still inconsistent with our intuitions regarding these counterfactuals. We do not merely have the 
intuition that it is not true that if $A_{n}$ were true, then either the past or the laws would be different; we also have the intuition that it is false.

The defender of violations might deny that there is a final world in the series before the world where the laws or the past are different, by instead holding that there are infinitely many worlds before the world where the laws or the past are different. Of course, this response cannot be appealed to in cases where the law only has finitely many instances. Moreover, it's not clear that this response can be applied to every instance of my argument for every law of nature, especially given the flexibility of the strategy of replacing talk of instances with talk of spacetime regions containing instances. In the absence of some reason to think that this strategy can be appealed to in every case, the defender of Violations is not justified in holding that there is never a final world in the series before the world where the past or the laws are different. At the very least, it is epistemically open on their view that there is a final world. In other words, it is epistemically open that there is a nomologically possible world where either the laws or the past are not weakly counterfactually robust. But this is enough to raise the challenge. If the intuition that the laws and the past are weakly counterfactually robust applies to the actual world, then it should apply to these worlds too.

It's natural to appeal to the violations themselves to explain why our intuitions don't carry over to these merely possible cases. The first thing to note is that it is implausible to hold that the existence of a single violation is enough to undermine our intuitions. First, this would undermine our knowledge of all counterfactuals with embedded antecedents, since each embedded antecedent involves adding a violation. Second, this even threatens to undermine our knowledge of counterfactuals without embedded antecedents, for if violations are possible, then we are not in a position to know that there are no violations. Consider a world exactly like our own, but containing 
a violation outside of our light cone. This is a relevantly similar world that our evidence does not rule out. If we cannot rule out this possibility, then we are not in a position to know that our world does not contain any violations, and so our knowledge of ordinary counterfactuals is undermined. But surely we do have knowledge of ordinary counterfactuals.

So the defender of violations should not hold that a single violation is enough to undermine our intuitions; our intuitions about counterfactuals are preserved when we add a single violation. Of course, this leaves open that our intuitions do not carry over to cases with large amounts of violations, which would be enough to defuse the problem of a nomologically possible world where the laws or the past are not weakly counterfactually robust. But if adding a single violation is not enough to undermine our intuitions, then it is puzzling why adding many violations does so. This is particularly puzzling because in the way I set up the argument, we are always moving forward in time. At each step of the argument, we are considering how the world would evolve differently in light of a new counterfactual supposition. So by construction, we are never considering how the future will evolve when that future involves violations. The violations are always prior to that future evolution. But it's hard to see why sufficiently many past violations would undermine our intuitions about how the world would evolve afterwards. If nothing else, this poses a substantial explanatory burden on the defender of Violations.

There's another problem with positing the possibility of violations in order to hold that the laws and the past are weakly counterfactually robust in the actual world, but then appealing to those violations in order to undermine our intuitions that the laws and the past are weakly counterfactually robust in a nomologically possible world. Suppose that after sufficiently many violations, the laws fail to be weakly counterfactually robust. But if we have the intuition that the laws are weakly counterfactually robust, then upon seeing that accumulating too many violations 
leads to the laws no longer being weakly counterfactually robust, rather than giving up our initial intuition it seems that we have a reason to deny that it is possible for that many violations to accumulate.

There's a final challenge for the defender of Violations arising from arbitrariness. As we add more violations, if the laws and the past are not always counterfactually preserved, at which point are they not preserved? Any particular point in the series of added violations seems arbitrary. There's nothing that picks out the point in the series that makes it especially salient for there to be a big shift in which counterfactuals are true. Moreover, there does not seem to be any reason to prefer which of either (3) or (4) fail. It's implausible that both would fail, but if there is no reason to prefer one or the other, then the view will be committed to making an arbitrary choice. This is especially worrying because an advantage of adopting Violations was supposed to be that it would enable us to avoid making a choice between holding that the past is counterfactually preserved and that the laws are counterfactually preserved.

Interestingly, the Humean about laws has a powerful response to this worry. BraddonMitchell may appeal to his best-systems account to explain the point at which there is a shift in the counterfactuals. Adding more violations will gradually change which system is the best system, and eventually a given law will no longer belong to the best system. Since adding violations will change which system is the best system, this explains why the laws are no longer counterfactually preserved, rather than the past. Moreover, since there will likely be no sharp point at which the laws change, there will also not be a sharp point at which the laws are no longer counterfactually preserved; rather both will be a vague matter. ${ }^{24}$

\footnotetext{
24 Of course, on an epistemicist view of vagueness, there will be a sharp point at which the laws change and are not counterfactually preserved, but we are unable to know where that point is. But since it is implausible that there is a sharp point at which the laws are no longer counterfactually preserved, the combination of Violations, Humeanism about the laws, and epistemicism about vagueness does not produce a very attractive package.
} 
But the non-Humean cannot make use of this response. To be clear, the non-Humean can hold that it is a vague matter at which point in the series the laws are no longer counterfactually preserved. But the non-Humean will not be able to explain why it is that the laws are no longer counterfactually preserved, rather than the past, and they will not be able to explain why some counterfactuals are borderline cases and others are not. It is the explanatory power that allows the Humean to avoid the arbitrariness worry. While the Humean can avoid the arbitrariness worry, the non-Humean cannot.

So we've seen reason to think that the prospects for defending Violations by appealing to the premise that the laws and the past are weakly counterfactually stable are not promising. Defenders of Violations are better off looking elsewhere for reason to accept Violations.

Acknowledgements Thanks to Phillip Bricker, Troy Cross, Patrick Grafton-Cardwell, Justis Koon, Chris Meacham, Alejandro Pérez Carballo, and two anonymous referees for very helpful comments and discussion. 


\section{References}

Armstrong, D. M. (1983). What is a Law of Nature. Cambridge: Cambridge University Press.

Beebee, Helen (2011). "Necessary Connections and the Problem of Induction." Nous 45, 3: 504-527.

Bennett, Jonathan (1984). “Counterfactuals and Temporal Direction.” Philosophical Review 93: 57-91.

Bird, Alexander (2007). Nature's Metaphysics. Oxford: Oxford University Press.

Braddon-Mitchell, David (2001). “Lossy Laws.” Nous 35, 2: 260-277.

Bricker, Phillip (1991). "Plenitude of Possible Structures.” Journal of Philosophy 88, 11: 607-619.

Carroll, John (1994). Laws of Nature. Cambridge: Cambridge University Press.

Cartwright, Nancy (1980). "Do the Laws of Physics State the Facts?" Pacific Philosophical Quarterly 61: 75-84.

Chalmers, David (1996). The Conscious Mind. Oxford: Oxford University Press. 
Dorr, Cian (2004). “Non-Symmetric Relations.” In Zimmerman (ed.), Oxford Studies in Metaphysics Volume 1. Oxford: Oxford University Press, 155-192.

-(2016). “Against Counterfactual Miracles.” Philosophical Review 125, 2: 241-286.

Dretske, Fred (1977). “Laws of Nature.” Philosophy of Science 44: 248-268.

Foster, John (1982-1983). "Induction, Explanation, and Natural Necessity." Proceedings of the Aristotelian Society 83: 87-101.

Goggans, Phillip (1992). “Do the Closest Counterfactual Worlds Contain Miracles?” Pacific Philosophical Quarterly 73: 137-149.

Hildebrand, Tyler (2018). "Natural Properties, Necessary Connections, and the Problem of Induction.” Philosophy and Phenomenological Research 96, 3: 668-689.

Huemer, Michael (2009). "Explanationist Aid for the Theory of Inductive Logic.” British Journal for the Philosophy of Science 60: 345-375.

Kment, Boris 2006. “Counterfactuals and Explanation.” Mind 115, 458: 261-309.

-2014. Modality and Explanatory Reasoning. Oxford: Oxford University Press. 
Lange, Marc 2000. Natural Laws in Scientific Practice. Oxford: Oxford University Press.

Lewis, David 1979. “Counterfactual Dependence and Time’s Arrow.” Nous 13: 455-476.

-1986a. "New Work for a Theory of Universals." Australasian Journal of Philosophy 61, 4: 343-377.

-1986b. On the Plurality of Worlds. Oxford: Blackwell.

Maudlin, Tim 2007. The Metaphysics within Physics. Oxford: Oxford University Press.

Roberts, John T. (2008). The Law-Governed Universe. Oxford: Oxford University Press.

Tooley, Michael 1977. “The Nature of Laws.” Canadian Journal of Philosophy 7: 667-698.

-2011. “The Skeptical Challenges of Berkeley and Hume: Can They Be Answered?" Proceedings and Addresses of the Philosophical Association 85, 2: 27-46. 